\title{
Reduced feedback designs for SDMA-OFDMA systems
}

\author{
Berna Özbek *, Didier Le Ruyet ${ }^{\dagger}$ \\ *Electrical and Electronics Engineering Department, Izmir Institute of Technology, Izmir, Turkey \\ Email: bernaozbek@iyte.edu.tr \\ $\dagger$ Electronics and Communications Laboratory, CNAM, Paris, France \\ Email: leruyet@cnam.fr
}

\begin{abstract}
In SDMA-OFDMA wireless communication systems, the feedback load increases with the number of users, subcarriers and antennas in the cell. In this paper, we propose two efficient reduced feedback algorithms by selecting the clusters at the user side. For each cluster, we select the users according to their norm and their orthogonality. We evaluate the performance of the user selection algorithms considering the quantization effect. We also design a specific codebook design to quantize CSI for the proposed criterion. ${ }^{1}$
\end{abstract}

\section{INTRODUCTION}

Orthogonal Frequency Division Multiple Access (OFDMA) and Space Division Multiple Access (SDMA) are promising technologies for the flexible high-rate services in future mobile radio networks since the large number of degrees of freedom in space, frequency, and time can be provided using channel state information (CSI) at the transmitter side [1]. The OFDMA is based on orthogonal frequency division multiplexing (OFDM) and inherits its superiority of mitigating multipath fading and maximizing throughput. The SDMA uses a beamforming technique in a multiple-antenna system and multiplexes multiple users on the same subcarrier to increase the spectral efficiency. For OFDMA systems, the sum data rate can be maximized when each subcarrier is assigned to the user with the best channel gain. For SDMA-OFDMA systems, the sum data rate can be maximized when the optimal set of co-channel users is selected for each subcarrier [2].

In order to exploit the full gain of the SDMA-OFDMA systems, the transmitter needs to know the CSI of all subcarriers, users and antennas. In time division duplexing (TDD) systems, CSI may be estimated at the transmitter using channel reciprocity, however, frequency division duplexing (FDD) systems lack of channel reciprocity. This problem can be overcome by sending the CSI from the receiver to the transmitter over a limited rate feedback channel. In this case, it is possible to use quantized CSI using a codebook that is known to both the transmitter and the receiver side [4]. The codebook design for narrowband single user communication system is a well studied problem[3]. The extension to the OFDM case has been considered in [5] [6]. In SDMA-OFDMA systems, the rate of the feedback link is high when all the users send their CSI. Besides that, a sophisticated resource allocation (RA)

\footnotetext{
${ }^{1}$ The work was partially supported by the Tubitak-PIA Bosphorus Project.
}

algorithm is needed to efficiently assign the resources to all the users.

For OFDMA systems, Svedman and et al. [7] have proposed to reduce the feedback load using the so-called clustered S-best criterion where adjacent subcarriers are grouped into clusters and only the CSI related to the strongest $\mathrm{S}$ clusters of each user are feedback to the transmitter.

For multiuser multiple-input single-output (MU-MISO) systems with linear precoding, we have proposed in [8] a semiorthogonal user selection algorithm to reduce the feedback load by combining the classical norm criterion with a criterion based on the orthogonality between the co-channel users.

In this paper, we propose an efficient algorithm to reduce the feedback load for SDMA-OFDMA systems. We firstly extend the clustered S-best criterion for SDMA-OFDMA systems supporting up to $N_{t}$ beams with adaptive feedback rate schemes. We use the cluster structure where the correlation is high between the subcarriers within the cluster so that the feedback of only one value is sufficient, e.g. CSI values belong to the weakest subcarrier of each antenna or the minimum subcarrier in terms of channel vector gain. Since the amount of feedback information needed from each user depends on the number of users in the system, we define the number of clusters for which each user will feedback their CSI adaptively according to the clustered S-best criterion. We propose to combine the clustered S-best and the semi-orthogonal user selection criterion for SDMA-OFDMA systems using zeroforcing (ZF) precoding. We propose a codebook design by quantizing CSI for the proposed criterion considering the properties of the clustered S-best and the semi-orthogonal criterion.

This paper is organized as follows. In section II, we describe the system model of SDMA-OFDMA over multipath wireless channels including the resource allocation algorithm. Then, we present the reduced feedback designs for SDMA-OFDMA systems considering clustered S-best and the proposed combined criterion in section III. The quantization of CSI for the proposed criterion is broached in section IV. In section V, we show the performance and comparison results. Section VI draws the concluding remarks of this paper. 


\section{SDMA-OFDMA SYSTEM MODEL}

We consider a SDMA-OFDMA system with $N_{t}$ transmit antennas, $K$ users and $N$ subcarriers. In this model, the channel vector between the base station and the $k$ th user for the $n$th subcarrier is described by

$$
\overline{\mathbf{H}}_{k, n}=\left[\begin{array}{llll}
\bar{H}_{k, n, 1} & \bar{H}_{k, n, 2} & \ldots & \bar{H}_{k, n, N_{t}}
\end{array}\right]
$$

where $\bar{H}_{k, n, t}$ is the channel information from the $t$ th transmit antenna to the $k$ th user for the $n$th subcarrier.

For a system where $N_{t} \leq K$, let $\mathcal{S}_{n}$ be the set of $N_{t}$ users scheduled at subcarrier $n$. The associated users' data are transmitted using ZF precoding [9]. Then $\overline{\mathbf{H}}\left(\mathcal{S}_{n}\right)$ denotes the matrix consisting of $N_{t}$ channel vectors of the selected users at subcarrier $n$. The relation between the data vector $\mathbf{S}_{n}\left(\mathcal{S}_{n}\right)$ and the received signal for $k$ th user and $n$th subcarrier can be written as:

$$
\begin{aligned}
Y_{k, n} & =\overline{\mathbf{H}}_{k, n} \mathbf{X}\left(\mathcal{S}_{n}\right)+N_{k, n} \\
& =\overline{\mathbf{H}}_{k, n} \overline{\mathbf{W}}\left(\mathcal{S}_{n}\right) \mathbf{P S}\left(\mathcal{S}_{n}\right)+N_{k, n}
\end{aligned}
$$

where $\mathbf{P}=\operatorname{diag}\left(\sqrt{P_{T} /\left(N N_{t}\right)}, \cdots, \sqrt{P_{T} /\left(N N_{t}\right)}\right)$ where the total transmit power $P_{T}$ is equally shared between the subcarriers and the beams, $\mathbf{X}\left(\mathcal{S}_{n}\right) \in \mathcal{C}^{N_{t} \times 1}$ is the transmitted symbol from the base station antennas, subject to a short-term power constraint (the transmitted signal must satisfy the power constraint $\left.\mathbf{X}\left(\mathcal{S}_{n}\right)^{H} \mathbf{X}\left(\mathcal{S}_{n}\right) \leq P_{T} /\left(N N_{t}\right)\right)$. The $\mathrm{ZF}$ transmit beamforming matrix is given by

$$
\overline{\mathbf{W}}\left(\mathcal{S}_{n}\right)=\alpha \overline{\mathbf{H}}\left(\mathcal{S}_{n}\right)^{H}\left(\overline{\mathbf{H}}\left(\mathcal{S}_{n}\right)^{H} \overline{\mathbf{H}}\left(\mathcal{S}_{n}\right)\right)^{-1}
$$

In order to keep the short term power constant, we have:

$$
\alpha=\frac{1}{\sqrt{\operatorname{tr}\left(\left(\overline{\mathbf{H}}\left(\mathcal{S}_{n}\right)^{H} \overline{\mathbf{H}}\left(\mathcal{S}_{n}\right)\right)^{-1}\right)}}
$$

In order to reduce the amount of feedback information and simplify the RA algorithm, we will group $N_{q}$ adjacent subcarriers in a cluster. Then, the precoding vector will be identical within a cluster of $N_{q}$ subcarriers. The SDMAOFDMA system with $N_{t}$ transmit antennas, $K$ users and $Q=N / N_{q}$ clusters is drawn in Figure 1 .

Our first objective is to optimize the cluster and beam allocation in order to maximize the sum capacity under the total power constraint equally shared between the clusters and the beams applying ZF precoding. Mathematically, the optimization problem considered in this paper is formulated as,

$$
\max _{\rho_{k, q, b}} R=\max _{\rho_{k, q, b}} \sum_{b=1}^{N_{t}} \sum_{k=1}^{K} \sum_{q=1}^{Q} \frac{\rho_{k, q, b}}{Q} \log _{2}\left(1+\mathbf{S I N R}_{k, q, b}\right)
$$

subject to

$$
\sum_{k=1}^{K} \rho_{k, q, b}=1 \quad \text { for each } q, b
$$

where

$\rho_{k, q, b}= \begin{cases}1 & k \text { th user is allocated to the beam } b \text { for cluster } q \\ 0 & \text { else }\end{cases}$

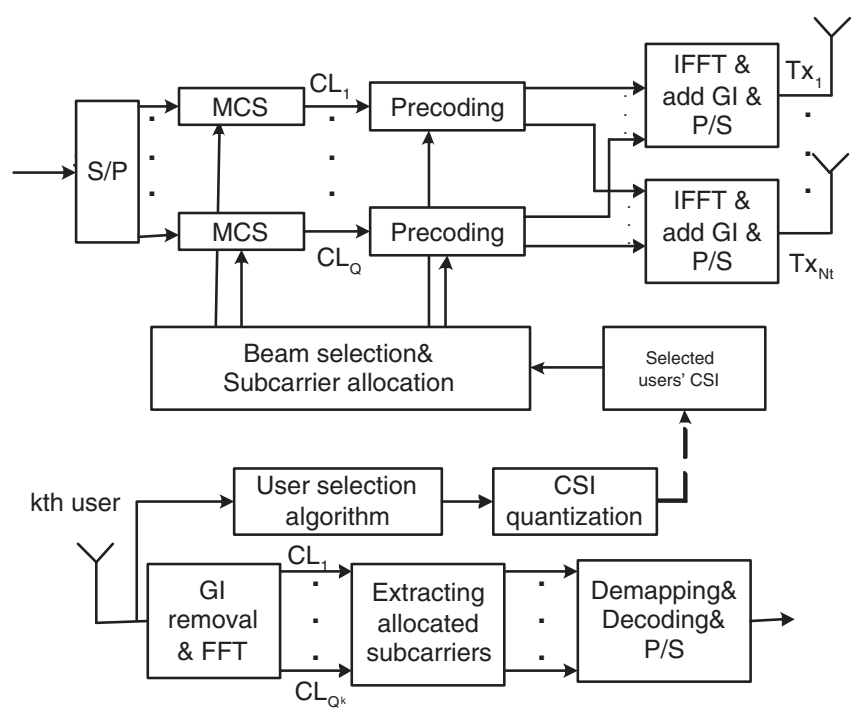

Fig. 1. The transmitter and kth receiver structure for SDMA-OFDMA system.

Consequently, only one user is allocated to each cluster and each beam.

The signal-to-interference-plus-noise-ratio (SINR) at user $k$ on $b$ th beam for $q$ th cluster can be calculated as:

$$
\mathbf{S I N R}_{k, q, b}=\frac{\frac{P_{T}}{N_{t}}\left|\mathbf{H}_{k, q} \mathbf{W}_{b}\left(\mathcal{S}_{q}\right)\right|^{2}}{N_{0} B_{w}+\frac{P_{T}}{N_{t}} \sum_{j=1 ; j \neq b}^{N_{t}}\left|\mathbf{H}_{k, q} \mathbf{W}_{j}\left(\mathcal{S}_{q}\right)\right|^{2}}
$$

where $N_{0}$ is the power spectral density of additive white Gaussian noise (AWGN) and $B_{w}$ is the total available bandwidth.

The precoding matrix, $\mathbf{W}\left(\mathcal{S}_{q}\right)$ calculated according to Equation (4), is defined by,

$$
\mathbf{W}\left(\mathcal{S}_{q}\right)=\left[\begin{array}{llll}
\mathbf{W}_{1}\left(\mathcal{S}_{q}\right) & \mathbf{W}_{2}\left(\mathcal{S}_{q}\right) & \ldots & \mathbf{W}_{N_{t}}\left(\mathcal{S}_{q}\right)
\end{array}\right]^{T}
$$

where $\mathbf{W}_{b}\left(\mathcal{S}_{q}\right)$ is the precoding vector for the $b$ th beam and the $q$ th subcarrier with the dimension of $N_{t} \times 1$.

The channel cluster vector $\mathbf{H}_{k, q}$ associated to the $k$ th user for the $q$ th cluster is given by:

$$
\mathbf{H}_{k, q}=\left[\begin{array}{llll}
H_{k, q, 1} & H_{k, q, 2} & \ldots & H_{k, q, N_{t}}
\end{array}\right]
$$

where $H_{k, q, t}$ is the channel information from the $t$ th transmit antenna to the $k$ th user for the $q$ th cluster. $\mathbf{H}_{k, q}$ can be calculated as follows:

$$
\mathbf{H}_{k, q}=\overline{\mathbf{H}}_{k, n^{\prime}}
$$

where

$$
n^{\prime}=\arg \min _{1 \leq i \leq Q}\left\{\left\|\overline{\mathbf{H}}_{k,(q-1) N_{q}+i}\right\|^{2}\right\}
$$

\section{Reduced Feedback Designs}

In order to exploit the multiuser diversity and maximize the sum capacity, the users with the highest supportable rate should be selected. However, this approach is unfair since the users with the lowest SINR will never be scheduled. To guaranty fairness, all the users should feedback the same 
amount of CSI. Nevertheless, the feedback rate can be reduced by letting each user sending their CSI to the base station associated only to a subset of clusters. In this paper, we will consider two criteria for the selection of these subsets for SDMA-OFDMA systems.

\section{A. V1:Clustered S-best Criterion}

For the Clustered S-best criterion, each user $k$ selects independently a set $\mathcal{S}_{k}$ composed of the $S_{x}$ clusters with the highest channel norm $\left\|\mathbf{H}_{k, q}\right\|$ as a extension of the work presented in [7] to the SDMA-OFDMA case. Then, each user feedbacks the CSI associated to the selected clusters to the base station. Let $\mathcal{T}_{q}$ be the set of users that have feedback their CSI associated to the cluster $q$ :

$$
\mathcal{T}_{q}=\left\{k \in\{1,2, \ldots, K\}: k \in \mathcal{S}_{k}\right\}
$$

Then, for each cluster $q$, the base station selects the set $\mathcal{S}_{q}$ to maximize the data rate. Since the total feedback rate is proportional to $K S_{x}$, it is reasonable to adjust $S_{x}$ in function of $K$ according to a desired function $S_{x}=f(K)$. This function is calculated offline according to a given target. In [7], the authors proposed to compute the function in order that the probability of less than $\alpha \%$ of the cluster can be assigned as $P_{\text {target }}$. This criterion can be extend to the multibeam case as follows:

Let introduce the indicator function $I_{q}^{N_{t}}$ :

$$
I_{q}^{N_{t}}= \begin{cases}1 & \text { if }\left|\mathcal{T}_{q}\right| \geq N_{t} \\ 0 & \text { else }\end{cases}
$$

The criterion becomes

$$
\operatorname{Pr}\left(\sum_{q=1}^{Q} I_{q}^{N_{t}} \leq \alpha Q\right)=P_{\text {target }}
$$

Another criterion can be to compute the desired function in order to reach $90 \%$ of the full CSI sum capacity.

\section{B. V2:Proposed Combined Criterion}

We propose to select the clusters according to the information on both the channel norm/quality and channel direction. For each cluster, firstly, we will select users whose channel directions are semi-orthogonal. Therefore, for each cluster we generate $N_{t}$ random orthonormal vectors $\phi_{i, q}\left(N_{t} \times 1\right)$ , $i=1, \ldots, N_{t}$. These vectors are the same for all the users but are independent between the clusters.

The users measure the orthogonality between their channels and the random vectors $\phi_{i, q}$ for each cluster using the chordal distance as:

$$
d^{2}\left(\mathbf{H}_{k, q}^{n o r m}, \phi_{i, q}\right)=1-\left|\mathbf{H}_{k, q}^{n o r m H} \phi_{i, q}\right|^{2}
$$

where $\mathbf{H}_{k, q}^{\text {norm }}=\frac{\mathbf{H}_{k, q}}{\left\|\mathbf{H}_{k, q}\right\|}$ is the normalized channel vector of the user $k$ and cluster $q$.

Let $\mathcal{O}^{N_{t}}$ be the unit sphere lying in $\mathcal{C}^{N_{t}}$ and centered at the original. Using the chordal distance metric, we can define a spherical cap on $\mathcal{O}^{N_{t}}$ with center $\mathbf{o}$ and square radius $\epsilon_{t h}$ as the open set:

$$
\mathcal{B}_{\epsilon}(\mathbf{o})=\left\{\mathbf{g} \in \mathcal{O}^{N_{t}}: d^{2}(\mathbf{g}, \mathbf{o}) \leq \epsilon_{t h}\right\}
$$

For the semi-orthogonal criterion, we have:

$$
\mathcal{T}_{q}^{\prime}=\left\{k \in\{1,2, \ldots, K\}: \mathbf{H}_{k, q} \in \bigcup_{i=1}^{N_{t}} \mathcal{B}_{\epsilon}\left(\phi_{i, q}\right)\right\}
$$

where $\mathcal{T}_{q}^{\prime}$ is the set of near orthogonal users for cluster $q$.

$\mathcal{T}_{k}^{\prime}$ is the set of the selected clusters by user $k$ :

$$
\mathcal{T}_{k}^{\prime}=\left\{q \in\{1,2, \ldots, Q\}: k \in \mathcal{T}_{q}^{\prime}\right\}
$$

Then, each user $k$ selects a set $\mathcal{S}_{k}$ of the $S_{x}$ best clusters from $\mathcal{T}_{k}^{\prime}$ in terms of channel norm. From the sets $S_{k}, k=1,2, \ldots, K$, we can construct the set $\mathcal{T}_{q}$ using Equation (13) for each $q$.

The choice of $\epsilon$ is critical since it directly effects the number of users for each cluster. The percentage of clusters that are semi-orthogonal according to proposed criterion is approximately calculated as $(2 \epsilon 100) \%$. Therefore, for each user, it is guaranteed that the proposed criterion is fulfilled by at least $S_{x}$ clusters when properly selecting the $\epsilon$ parameter .

\section{QuAntization OF THE CSI}

In order to transmit the CSI from the users to the base station, a classical solution is to quantize the channel direction information (CDI) and the channel quality information (CQI) before transmission over the finite rate feedback link. In [10], the Lloyd algorithm was suggested for the design of the vector codebook. The codebook should be constructed by minimizing the maximum inner product between codewords and this results in the Grassmannian line packing solution when the channel vector is independent identically distributed (i.i.d).

The quantized normalized channel vector $\mathbf{H}_{b}^{\text {norm }}\left(\mathcal{S}_{q}\right)$ associated to the $b$ th beam and $q$ th cluster is taken from a set of $2^{F}$ vectors where $F$ is the number of feedback bits.

\section{A. Proposed codebook design}

In contrast to the normalized i.i.d channel isotropically distributed in $\mathcal{O}^{N_{t}}$, when we are using the semi-orthogonal criterion, the clusters are selected according to a spherical cap region. Consequently, it is important to build the codebook using a quantization of the localized region or local packing. A local Grassmannian packing with parameters $N_{t}, \mathbf{o}, \epsilon_{t h}$ is a set of $N_{c}$ vectors, where $N_{c}$ is the codebook size, $\mathbf{g}_{i}$, $i=1, \ldots, N_{c}$, constrained to a spherical cap $\mathcal{F}_{\epsilon}(\mathbf{o})$ in $\mathcal{O}^{N_{t}}$ such that

$$
\min _{1 \leq i<j \leq N} d^{2}\left(\mathbf{g}_{i}, \mathbf{g}_{j}\right)
$$

is maximized. As in the i.i.d. case, we use vector quantization to design these local packings.

For the semi-orthogonal criterion, the codebook must be adapted according to the orthogonal vectors $\phi_{i, q}$. From the local packing associated to the spherical cap $\mathcal{F}_{\epsilon}(\mathbf{o})$, it is possible to compute the local packing associated to the spherical cap $\mathcal{F}_{\epsilon}(\mathbf{o})$ using the rotation matrix. 


$$
\phi_{i, q}=\mathbf{U}_{\text {rot }} \mathbf{o}
$$

where $\mathbf{U}_{\text {rot }}$ is the unitary rotation matrix.

When the user CDI is inside the spherical cap region, the user will feedback $\log _{2}\left(N_{c}\right)$ bits corresponding to the codebook index. In addition to that, it will be necessary to feedback $\log _{2}\left(N_{t}\right)$ bits corresponding to the index of the vector $\phi_{i}$. Consequently, for a codebook size $N_{c}, \log _{2}\left(N_{c} \times N_{t}\right)$ bits will be necessary to quantify the CDI.

Although the selection of clusters using the semi-orthogonal criterion doesn't offer any gain when the CSI is not quantized, thank to the spherical cap, the quantization error of the CDI is much lower than using the classical quantization.

\section{PRoportional FAIRnESS FOR SDMA-OFDMA}

Since we assume that the users are distributed heterogeneously, we choose to use the proportional fairness (PF) algorithm to allocate users by adding a set of nonlinear constraints. This scheduler brings a compromise between the sum capacity and the fairness. The fairness index (FI) is calculated by using Jain index [11] given as:

$$
\mathrm{FI}=\frac{\left(\sum_{k=1}^{K} R_{k}\right)^{2}}{K \sum_{k=1}^{K} R_{k}^{2}}
$$

where $R_{k}$ is the user capacity. The FI ranges between 0 (no fairness) and 1 (perfect fairness) in which all users would achieve the same data rate.

In order to allocate the users to the clusters and beams fairly, we propose PF algorithm as following:

For each cluster $q$ :

We can construct $\left(\begin{array}{c}\left|T_{q}\right| \\ N_{t}\end{array}\right)$ set of $\mathcal{S}_{q}^{i}$ of size $N_{t}$ from the set of $\mathcal{T}_{q}$.

For each set $\mathcal{S}_{q}^{i}$ :

- Calculate the capacity of each user $k^{\prime}(b)$ associated to the $b$ th beam within the set $\mathcal{S}_{q}^{i}$ obtaining from the set $\mathcal{T}_{q}$ : For each $b$ :

$$
C_{k^{\prime}(b), q, i}=\log _{2}\left(1+\frac{\left(P_{T} / N_{t}\right)\left|\mathbf{H}_{k^{\prime}, q} \mathbf{W}_{b}\left(\mathcal{S}_{q}^{i}\right)\right|^{2}}{N_{0} B_{w}}\right)
$$

End.

- Choose the $S_{q}$ user set to transmit from the $q$ th cluster as

$$
j=\arg \max _{i} \sum_{b=1}^{N_{t}} \frac{C_{k^{\prime}(b), q, i}}{T_{p, k^{\prime}(b)}}
$$

Then, we obtain $\mathcal{S}_{q}=\mathcal{S}_{q}^{j}$.

- Update the capacity of the selected users given in $\mathcal{S}_{q}$. End.

Then, update the filtered capacity, $T_{p, k}$ at time $p$, for each user $k$ :

$$
T_{p, k}=\left(1-\frac{1}{t_{c}}\right) T_{p-1, k}+\frac{1}{t_{c}} \bar{C}(p, k)
$$

where $\bar{C}(p, k)$ is the effectively allocated capacity to user $k$ at time $p$.

\section{Vi. Performance Evaluation}

We perform the simulation results to illustrate the benefits of the reduced feedback user selection algorithms in a single-cell SDMA-OFDMA system. The users are uniformly distributed in the cell area and the simulation parameters are presented in the Table I.

TABLE I

THE SYSTEM PARAMETERS FOR SIMULATION PERFORMANCE

\begin{tabular}{||c||c||}
\hline \hline \multicolumn{1}{||c||}{ Parameter } & Value \\
\hline \hline Cell radius & $1600 \mathrm{~m}$ \\
\hline BS Transmit Power & $43.10 \mathrm{dBm}$ \\
\hline Noise Power & $-174 \mathrm{dBm}$ \\
\hline Path Loss $L_{p}$ & $128.1+37.6 \log _{10}(d) \mathrm{dB}$ \\
\hline Channel Model & $3 \mathrm{GPP}, \mathrm{TU}$ \\
\hline Number of clusters & 48 \\
\hline Bandwidth & $10 \mathrm{MHz}$ \\
\hline Carrier frequency & $2.4 \mathrm{GHz}$ \\
\hline Velocity & $3 \mathrm{~km} / \mathrm{h}$ \\
\hline Simulation Time & $10 \mathrm{~s}$ \\
\hline \hline
\end{tabular}

In order to compare the performance of the proposed designs, we show the sum capacity results of the quantized reduced feedback based on V1 and proposed V2 criterion. Before that, to define the feedback load, we should show the effect of number of selected clusters on the performance to adjust the sufficient adaptive feedback rate based on the number of users in the system.

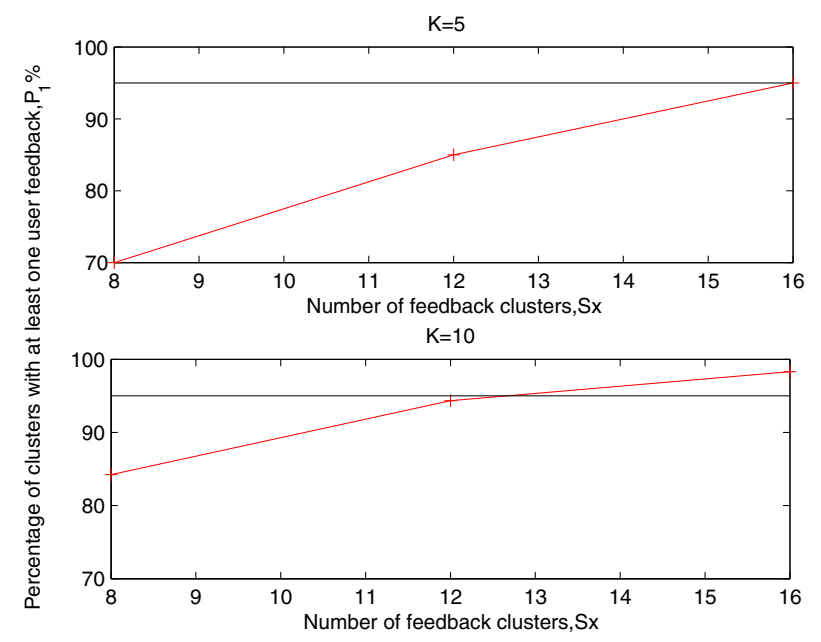

Fig. 2. Percentage of clusters with at least one user feedback, $P_{1} \%$ versus the number of feedback clusters $S_{x}$ for $K=5$ and 10.

According to the percentage of clusters with at least one user feedback shown in Figure 2 and 3, the number of selected clusters is chosen to satisfy the $95 \%$ of the fulfilled ratio for different $K$ values. Then, the corresponding $\epsilon$ value is defined as $2 \epsilon Q$ is equal or higher than $S_{x}$ to guarantee that at least $S_{x}$ clusters are selected for each user. All these parameters required for the SDMA-OFDMA reduced feedback designs are listed in Table II. 


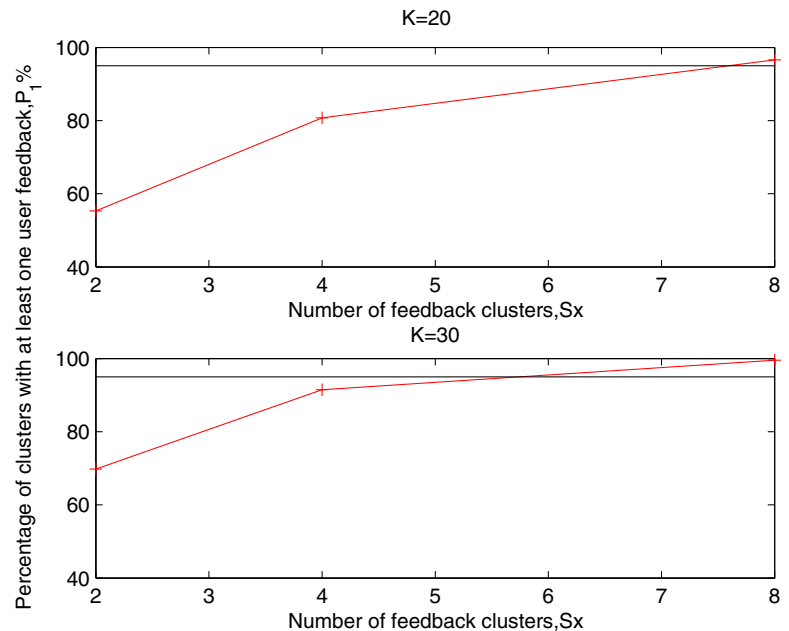

Fig. 3. Percentage of clusters with at least one user feedback, $P_{1} \%$ versus the number of feedback clusters $S_{x}$ for $K=20$ and 30 .

TABLE II

THE PARAMETERS OF THE REDUCED FEEDBACK DESIGNS

\begin{tabular}{|c||c|c|c|c|}
\hline \hline$K$ & 5 & 10 & 20 & 30 \\
\hline$S_{x}$ & 16 & 12 & 8 & 6 \\
\hline$\epsilon$ & 0.25 & 0.2 & 0.15 & 0.125 \\
\hline$\left(P_{1} \%, P_{2} \%\right)$ & $(86,54)$ & $(94,75)$ & $(96,85)$ & $(98,87)$ \\
\hline \hline
\end{tabular}

In the Table II, $P_{1}$ and $P_{2}$ are the parameters that indicate the percentages of the number of the clusters with at least one and two users respectively feedback the CSI to the base station for a given $S_{x}$.

According to the parameters given in Table I and II, the performance results for the V1 criterion and the proposed V2 criterion are shown in Figure 4 and 5, respectively. $Q$ and $D$ represent the number of feedback bits for CQI and CDI respectively. We can observe that the quantization of the channel quality information (CQI) has no impact on the performance for both criteria since $Q 4 D 4, Q 6 D 4$ and $Q 8 D 4$ give almost the same sum capacity performances. The similar behavior is also observed for the case of $C Q I=6$ and 8 . Therefore, we can conclude that only feedback of CDI to the base station is enough.

In Figure 6, the proposed V2 criterion and the V1 criterion are compared for SDMA-OFDMA systems. According to the results, the proposed algorithm outperforms the clustered Sbest criterion. Since the SDMA-OFDMA systems are sensitive to interference, it is important to minimize the CDI quantization error. We can reduce the quantization error of the CDI using the proposed codebook design. Consequently the sum capacity performance of proposed V2 algorithm is much higher than V1 criterion. Moreover, when the number of CDI quantization bits decreases, the performance difference between the two criteria increases.

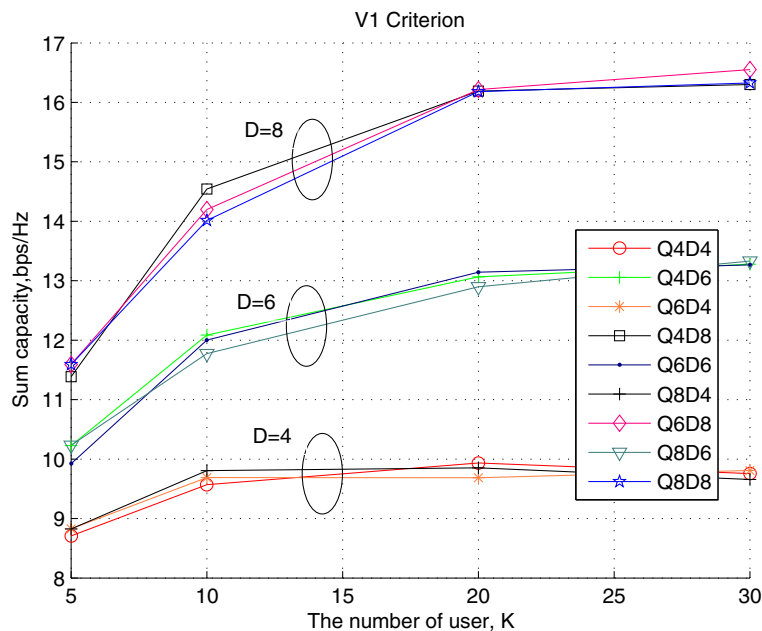

Fig. 4. Sum data rate versus number of users for Clustered S-best with quantized feedback.

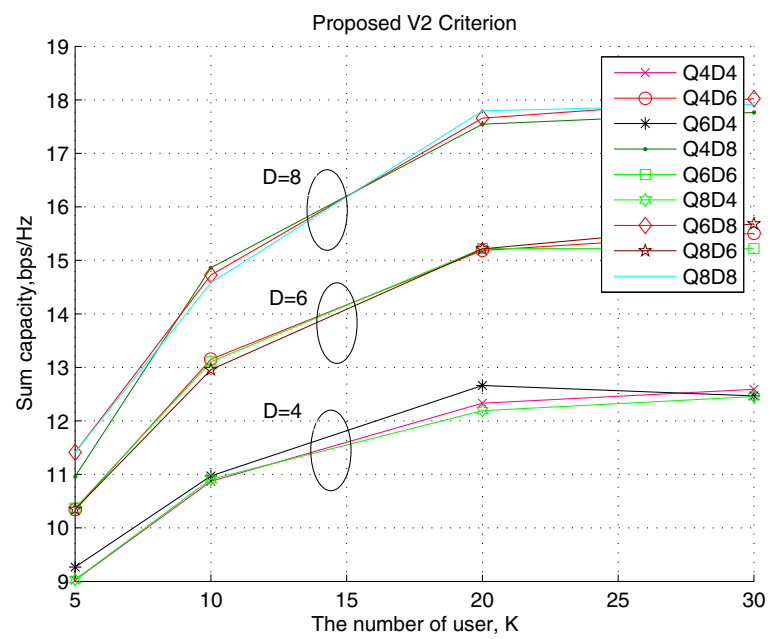

Fig. 5. Sum data rate versus number of users for proposed algorithm with quantized feedback.

\section{CONCLUSION}

In this paper, we have proposed and evaluated two efficient algorithms to reduce the feedback load for SDMA-OFDMA systems considering quantization. The first one is the clustered S-best criterion that selects the feedback cluster according to their norm. The second one is the proposed combined method that selects the users according to their orthogonality and norm. For both criteria, the amount of feedback information is fixed. For the proposed criterion, since the users are selected according to a main direction, it is possible to build the CDI codebooks using a local packing. Thanks to the smaller CDI quantization error obtaining proposed codebook design, we have shown that the proposed scheme outperforms the clustered S-best criterion. Indeed, in an interference limited system, it is quite important to minimize the CDI quantization error. 


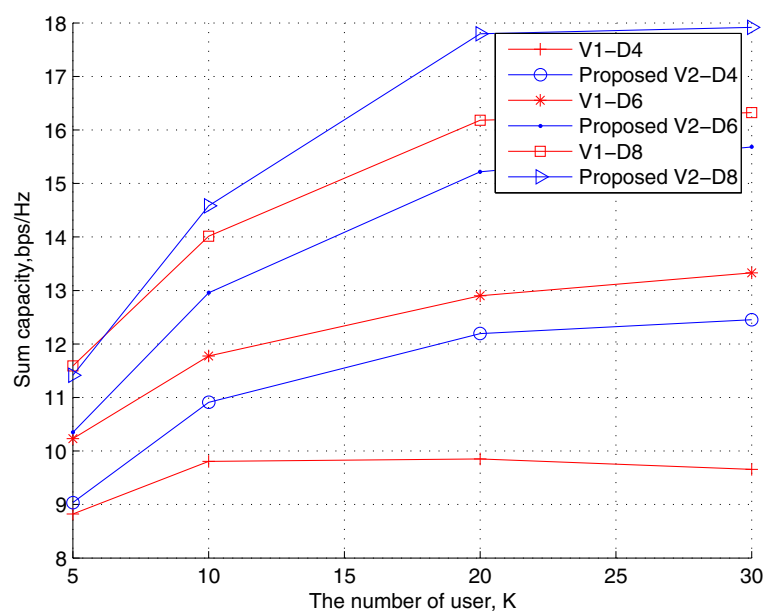

Fig. 6. Sum rate versus number of users for comparison with quantized feedback.

\section{REFERENCES}

[1] Y.J. Zhang and K. B. Letaif, "An efficient resource allocation scheme for spatial multiuser access in MIMO/OFDM systems", IEEE Trans. on Communications, vol. 53, pp.107-116,Jan. 2005.

[2] T.F. Maciel and A.Klein, "A resource allocation strategy for SDMA/OFDMA systems", Proc. of 16th IST Mobile and Wireless Communications Summit, pp.1-5, July 2007.

[3] S. Zhou, Z. Wang, and G. Giannakis, "Performance Analysis for Transmit-Beamforming with Finite-Rate Feedback", Proc. of 38th Conf. on Info. Sciences and Systems, Princeton Univ., NJ, March 17-19, 2004.

[4] D. J. Love, R. W. Heath, Jr., V. K. N. Lau, D. Gesbert, B. D. Rao, and M. Andrews, An Overview of Limited Feedback in Wireless Communication Systems, accepted to IEEE Journal on Selected Areas in Communications, 2008.

[5] J. Choi and R. W. Heath, Jr., "Interpolation based transmit beamforming for MIMO- OFDM with limited feedback," IEEE Trans. Sig. Proc., vol. 53, pp. 4125-4135, Nov. 2005.

[6] T. Pande, D. J. Love, J. V. Krogmeier, "Reduced Feedback MIMOOFDM Precoding and Antenna Selection", IEEE Transactions on Signal Processing vol. 55(5-2), pp. 2284-2293, may 2007.

[7] P. Svedman, S. K. Wilson, L. J. Cimini and B. Ottersten, "Opportunistic Beamforming and Scheduling for OFDMA systems", IEEE Trans. on Communications, vol. 55, pp.941-952,May 2007.

[8] D. Le Ruyet, H. Khanfir and B. Ozbek, "Performance of Semi-orthogonal user selection for Multiuser MISO systems", Proc. of IEEE Spawc, pp.June 2008, Brazil.

[9] T. Yoo and A. Goldsmith, "On the optimality of multiantenna broadcast scheduling using zero-forcing beamforming", IEEE Journal on Sel. Areas in Commun. (JSAC), vol. 24, no. 3, pp.528-541, Mar. 2006.

[10] A. Narula, M. J. Lopez, M. D. Trott, G. W. Wornell, "Efficient use of side information in multiple antenna data transmission over fading channels", IEEE Journal on Sel. Areas in Commun. (JSAC), pp.1423-1436, Oct. 1998.

[11] R. Jain, D.M. Chiu, W.R. Hawe, "A Quantitative Measure of Fairness and Discrimination for Resource Allocation Shared Computer Systems", Digital Equipment Corporation technical report TR-301, 1984. 